\title{
The Emergency Rush Repair Handling Design of a Certain Type Special Operation Computer
}

\author{
Liu Junxian ${ }^{1, a}$, Zhang Wei ${ }^{1, b}$, Wang Dawei ${ }^{1, c}$, Lou Xiaofang ${ }^{1, d}$ and Xiao Hang ${ }^{1, d}$ \\ ${ }^{1}$ Wuhan Mechanical Technology College, Wuhan, Hubei, China \\ a83950280@qq.com, b1224225295@qq.com, 416087321@qq.com, dugufei_2000@163.com
}

Keywords: Maintenance and support. Emergency handling. Rush repair handling.

Abstract. The stereotype and long-time usage of a special operation computer lead to frequent glitches, great difficulty in troubleshooting and maintenance and support, and so on. Because of its key status and role, it is necessary to make emergency rush repair of it. Therefore, in this paper, based on the study of the computer, the fault emergency repair process is designed, and the motherboard failure emergency treatment and machine failures emergency treatment of which are designed as well, including the required emergency repair equipment of these two programs, safeguarding the emergency repair needs of this operation computer.

\section{Introduction}

A certain type of computer is a special one, different from the ordinary computers. It is the core equipment of the information systems, which has high cost and a critical status. On the one hand, it receives the job scheduling and job instruction from the upper level, or reports job completion to the upper level; on the other hand, it control for control each job unit at the next level, issuing job instruction, job data and collecting job equipment consumption, job scheduling and job instruction execution and so on. However, this type of computer is with a longer stereotype and there is great difficulty in its maintenance and support, one of the major problem is that the electronic equipment after years of use is in the old age in their life cycle, failure-prone; the second main reason, owing to the rapid development and updates of computer technology, computer chip and device designed and selected many years ago has been discontinued, difficult to find in the market, the fault is difficult to rule out. The above makes a severe test for the maintenance and support of the computer. In order to keep the job continued when the computer fails, this paper designed the emergency repair process for this type of computer (two programs) as an alternative after the failure, and the corresponding products were developed to replace the running of original computer motherboard when in emergency treatment.

\section{System Prototype}

Original Computer Function. This type of job computer is dedicated, on the one hand, it runs its dedicated business software, with the functions of formulating work plans, work programs, generating a job command and other operations functions. On the other hand, it has functions of wired and wireless data-transmission communication and word-transfer communication. As well, it has three analog two-wire communication port, which can simultaneously communicate with the upper, lower and neighboring units by wired / Phone Fax. The original computer can analog wireless transmission, through data multiplexing equipment to assist digital wireless transmission equipment, and can transmit wireless digital data to the upper, lower and neighboring units.

Original Computer Components. This type of computer consists of motherboard, LVDS converter board, communication board, LCD display, power panel, pressure plate, keyboard panel, built-in lithium and interface boards and other components, as shown in Fig.1. 


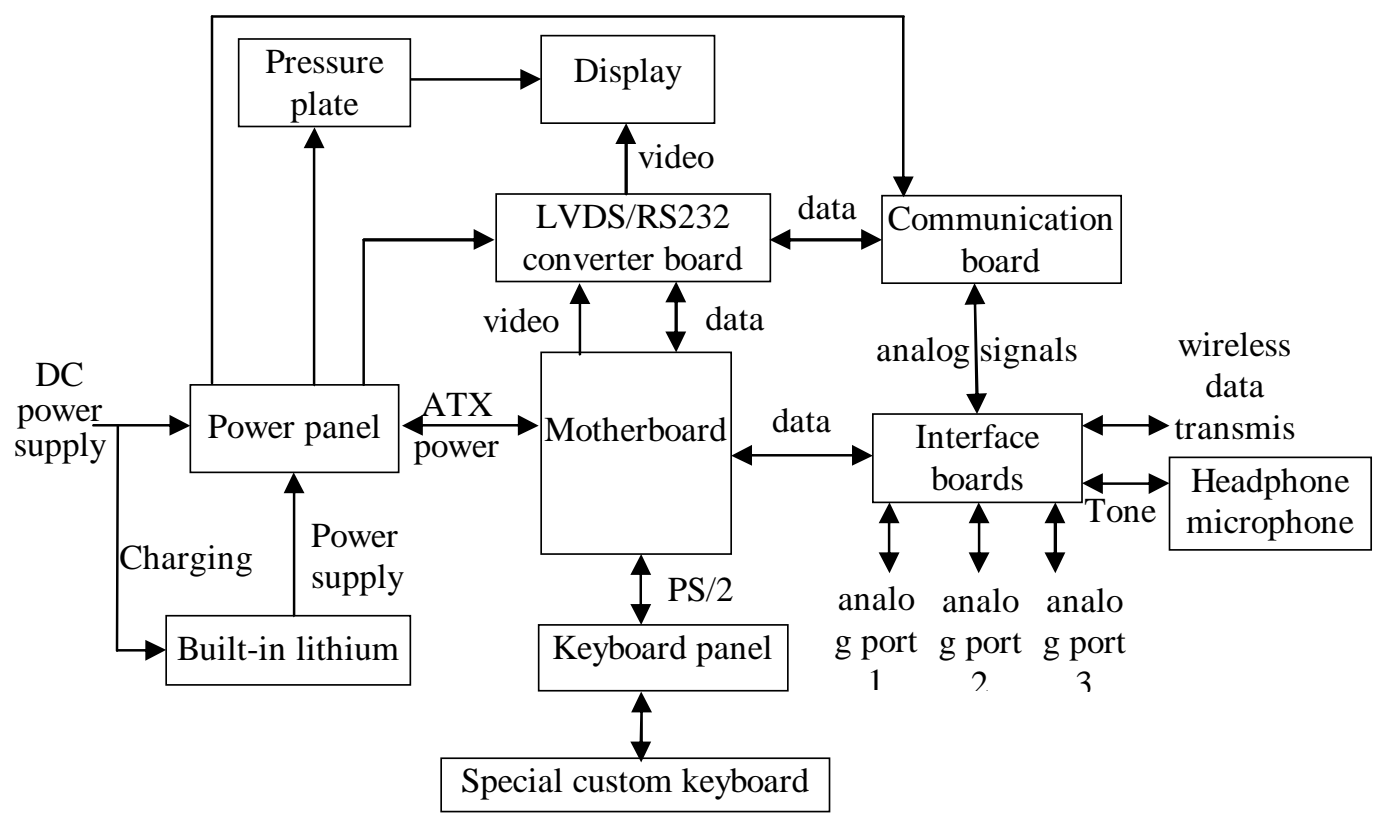

Fig.1. Block diagram of the original computer

Among them, the motherboard and communication board are the core components, which integrates chipset, CPU, memory, and electronic disk. Its showing output is 18bit RGB digital signal, and must be converted into LVDS format interfaced with LCD by LVDS converter board[1]. The wireless data transmission, through the motherboard COM2 port, connects directly with the interface board and wireless data transmission equipment. In the analog of wired or wireless transmission, motherboard COM1 port converts RS232C into TTL via LVDS converter board, and then reaches the communication board. After the process of modulation, amplification, 2/4-wire conversion through the communication board, three analog ports will be accessed to copper transmission lines to operate the transmission. LVDS converter board is to complete the conversion of RGB digital video signal to LVDS signal, and the conversion of communication data RS232C level to TTL level from the motherboard to communication board[2].

\section{System Design}

Emergency Handling Design of Motherboard Failure. For the above functions and composition of this type of computer, if the computer fails (which is also the reason of highest rate failure and hardest part in troubleshooting), according to the reasons mentioned above, the fault may not be ruled out, especially in the short time. In emergency repair, a motherboard emergency board shall be designed with the same functionality, power and size as the original motherboard, the communication interface is basically the same as the original as well. When in emergency repair case, mounting this circuit board on will replace the original board to work. After the design, the dedicated job software will be migrated to emergency treatment board, the emergency handling board will be mounted inside the computer chassis, under the condition of making no changes to other parts to replace the original board, shown in Fig. 2.

There are two differences from original motherboard in running. First, the output video of original motherboard is RGB 18bit digital signal, the board emergency board outputs directly LVDS signal, can contacted with the LCD display directly, thereby bypassing the LVDS converter board. Second, the original motherboard uses ATX power supply, motherboard emergency board directly uses $+12 \mathrm{~V}$ and $+5 \mathrm{~V}$ dual supply. Due to the motherboard plate power emergency power is consistent with the original motherboard, the motherboard plate may use $+12 \mathrm{~V}$ and $+5 \mathrm{~V}$ dual supply of ATX power supply output by the emergency power supply board. In addition, the power supply board is the DC / ATX power supply board, its PSON signal in ATX interface should have provided by the power management circuit board, but when the PSON of this power board suspended, the default is low level. Therefore, 
the motherboard plate can be turned on without providing PSON signal to the emergency power supply board, while at shutdown, it cannot provide a high level of PSON signal to soft-off, but which does not affect other functions[3].

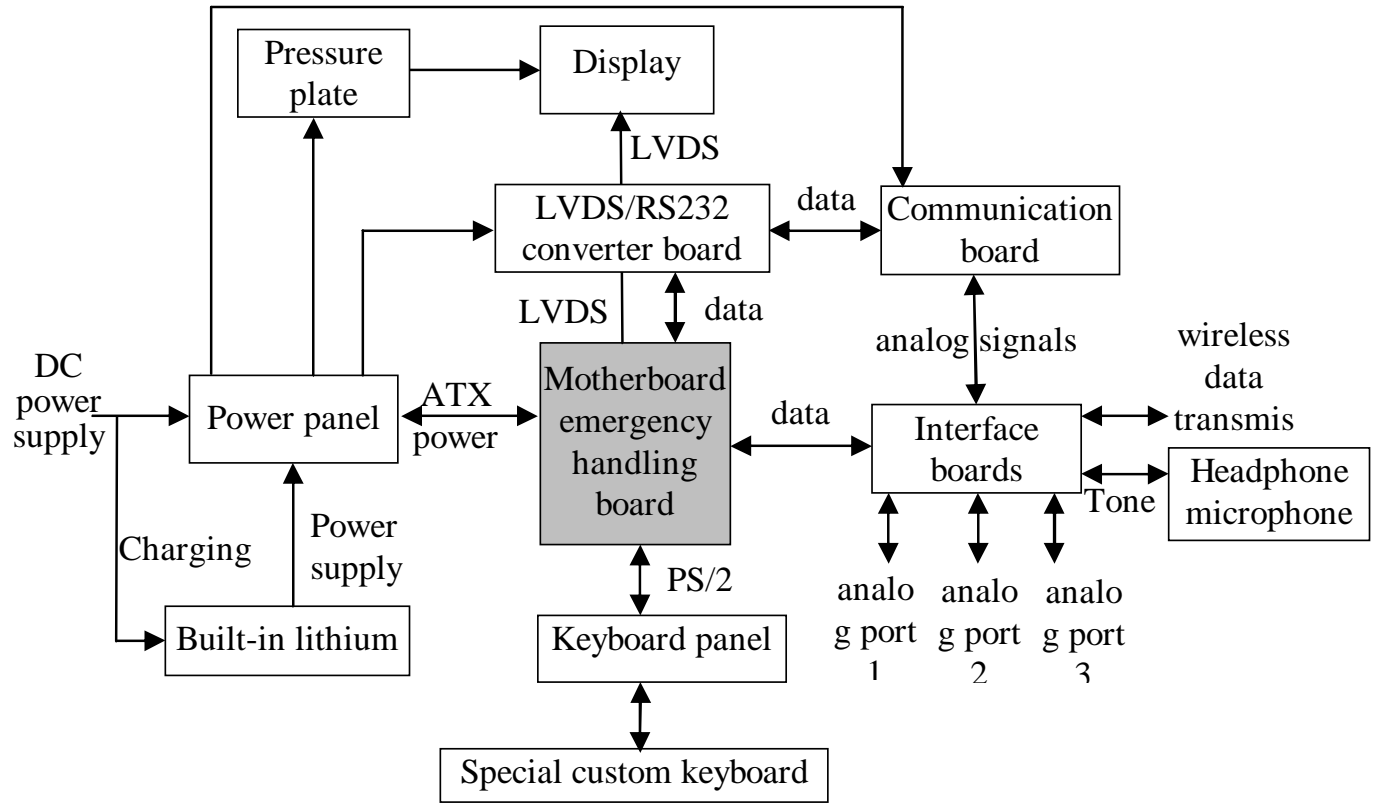

Fig. 2. Block diagram of the original computer

Emergency Handling Design of Machine Fault. If there are other components in fail except the computer motherboard, resulted in the troubleshooting unresolved and function recovery as soon as possible, the machine can be used as an alternative method for emergency treatment. Based on the design of emergency handling board, the design of this program works to use the original computer communication board, as shown in Fig. 3. The machine emergency handling board in the figure below is designed by this paper, communication board is the internal part of the original computer, after connecting to a standard monitor and a standard keyboard, it can complete the same job as the original computer.

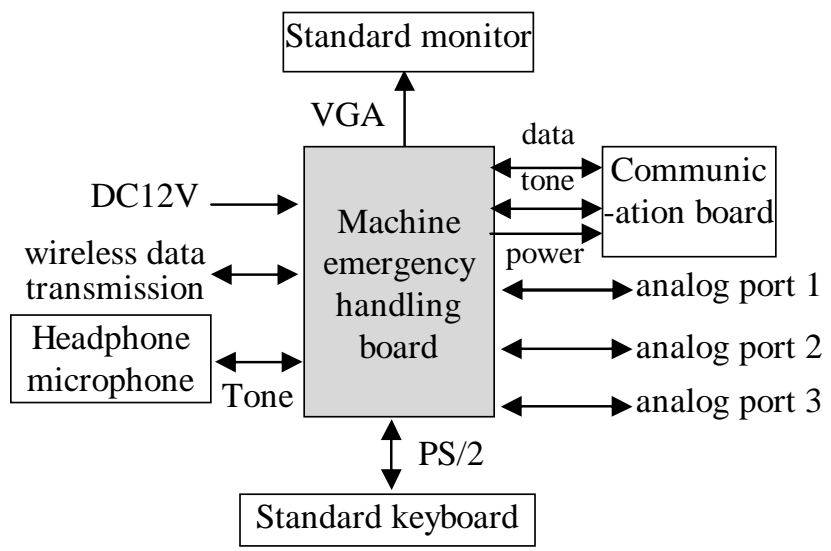

Fig. 3 the emergency handling plan of machine fault

Wherein, the composition of whole machine emergency panel is shown in Fig. 4. Machine emergency handling board integrates the power board, LVDS conversion and interface board of the original computer, increases the detection node and elicits each signal of communication. The detection of each node can proceed in the work, but also can make on-line testing from the front and the back of the communication board.

Among them, $12 \mathrm{~V}-5 \mathrm{~V}$ circuit mainly completes DC12V to DC5V power supply, which facilitates the core circuit board and the communication board to work. RS232-TTL circuit accomplishes the conversion from RS232 to TTL level in LVDS converter board of the original computer, realizing sending and receiving data channel from core board to communication board. Voice switching circuit achieves that a headset microphone switches respectively with analog $1,2,3$ ports to carry the voice 
communication. The interface circuit board completes communication with three analog ports, at the same time elicits the detection node. The detection signal elicitation of each signal realizes the on-line signal detection in case of operation. Core board circuit is basically the same as the motherboard emergency handling board, including CPU, chipset, memory, and disk and others, which is the basic platform of private job software, but also the core of the whole emergency board.

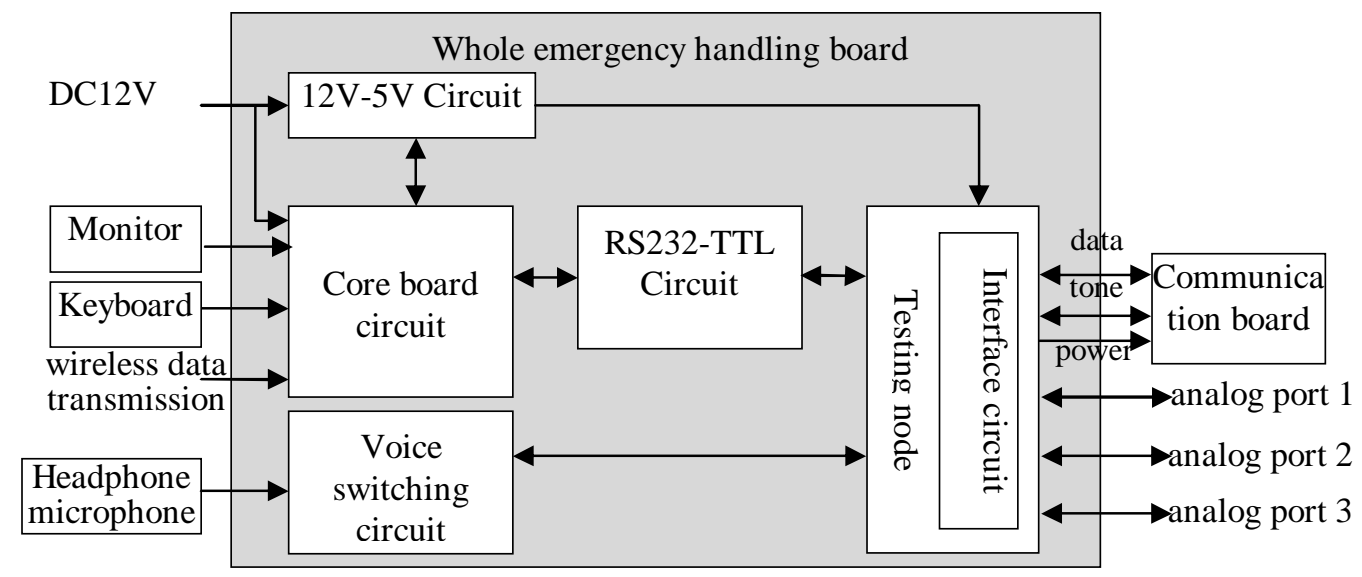

Fig. 4 Block diagram of the whole emergency handling board

\section{Summary}

Through the design of emergency repair of motherboard failure to this type of job computer machine and the emergency repair process design of whole machine failure, this paper developed motherboard emergency handling board and emergency board of whole machine respectively, realizing replacement of emergency repair process in case of motherboard failure alone, and the alternative emergency repair process in multi-component failure mode of the whole machine. By applying to the practice, this paper sets contingency plans at low cost and high efficiency method to handle the emergency failures of this type of computer. After the emergency repair process, these two alternative designs realized the same functions as the original job computer. Practice has proved that two contingency plans in this paper are successful and effective.

\section{References}

[1] Zhang Jian, Wu Xiaobing, The technology theory and design introduction of LVDS, forth ed., Application of Electronic Technique, 26 (2000) 24-28.

[2] Zhang Yigang, The theory and interface technology of Single Chip Microcomputer, People's Posts and Telecommunications Press, Beijing, 2011, pp. 178-180.

[3] Li Wengbing, The comprising theory of computer, forth ed., Tsinghua University Press, Beijing, 2010, pp. 155-161. 